\title{
Audiovestibular Symptoms in Systemic Autoimmune Diseases
}

\author{
Massimo Ralli $\mathbb{D},{ }^{1}$ Vittorio D'Aguanno $\mathbb{D},{ }^{2}$ Arianna Di Stadio $\left(\mathbb{D},{ }^{3}\right.$ Armando De Virgilio $\mathbb{D},{ }^{4}$ \\ Adelchi Croce, ${ }^{5}$ Lucia Longo, ${ }^{2}$ Antonio Greco $\mathbb{D D}^{2}$, and Marco de Vincentiis $\mathbb{D}^{1}$
}

${ }^{1}$ Department Oral and Maxillofacial Sciences, Sapienza University of Rome, Rome, Italy

${ }^{2}$ Department of Sense Organs, Sapienza University of Rome, Rome, Italy

${ }^{3}$ San Camillo Hospital IRCCS, Venice, Italy

${ }^{4}$ Humanitas Clinical and Research Center, Via Alessandro Manzoni 5620089 Rozzano, Italy

${ }^{5}$ Department of Otolaryngology, University G. D'Annunzio of Chieti-Pescara, Chieti, Italy

Correspondence should be addressed to Arianna Di Stadio; ariannadistadio@hotmail.com

Received 29 December 2017; Revised 11 June 2018; Accepted 4 July 2018; Published 19 August 2018

Academic Editor: Eirini Rigopoulou

Copyright ( 92018 Massimo Ralli et al. This is an open access article distributed under the Creative Commons Attribution License, which permits unrestricted use, distribution, and reproduction in any medium, provided the original work is properly cited.

\begin{abstract}
Immune-mediated inner ear disease can be primary, when the autoimmune response is against the inner ear, or secondary. The latter is characterized by the involvement of the ear in the presence of systemic autoimmune conditions. Sensorineural hearing loss is the most common audiovestibular symptom associated with systemic autoimmune diseases, although conductive hearing impairment may also be present. Hearing loss may present in a sudden, slowly, rapidly progressive or fluctuating form, and is mostly bilateral and asymmetric. Hearing loss shows a good response to corticosteroid therapy that may lead to near-complete hearing restoration. Vestibular symptoms, tinnitus, and aural fullness can be found in patients with systemic autoimmune diseases; they often mimic primary inner ear disorders such as Menière's disease and mainly affect both ears simultaneously. Awareness of inner ear involvement in systemic autoimmune diseases is essential for the good response shown to appropriate treatment. However, it is often misdiagnosed due to variable clinical presentation, limited knowledge, sparse evidence, and lack of specific diagnostic tests. The aim of this review is to analyse available evidence, often only reported in the form of case reports due to the rarity of some of these conditions, of the different clinical presentations of audiological and vestibular symptoms in systemic autoimmune diseases.
\end{abstract}

\section{Introduction}

The inner ear has been considered for a long time an immune-privileged site, spared from organ-specific autoimmunity and rarely involved in systemic autoimmune diseases thanks to the blood-labyrinthine barrier [1]. Lehnhardt [2] was the first to hypothesize that sudden or rapidly progressive sensorineural hearing loss (SNHL) could be the result of an autoimmune process against the inner ear. McCabe [3] showed the success of steroid and cytotoxic treatment in a cohort of patients with bilateral progressive SNHL, suggesting an autoimmune pathogenesis. Recently, several studies showed inflammatory cells in the inner ear, describing the presence of resident cochlear macrophages in animal models and the recruitment of inflammatory macrophages to the cochlea [4]. In 2016, O’Malley et al. identified cells with staining characteristics and morphology consistent with macrophages/microglia in the human cochlea [4]; the presence of these cells in patients with autoimmune diseases suggests that they may have an important role in inner ear pathology due to the increased level of proinflammatory cytokines and reactive oxygen species (ROS) induced by microglia [4].

There is growing interest for inner ear involvement in systemic autoimmune diseases $[5,6]$; this condition should be considered in patients with audiovestibular dysfunction presenting a constellation of symptoms consistent with systemic autoimmunity or with a preexisting diagnosis of autoimmune disease $[7,8]$. Inner ear involvement in systemic autoimmune diseases should be distinguished from primary autoimmune inner ear disease, a condition in which the immune response acts directly against the inner ear $[6,7]$. 
Inner ear involvement in autoimmune diseases is estimated to account for less than $1 \%$ of all cases of acquired hearing loss [7] and follows gender and demographic characteristics of autoimmune disorders, with higher prevalence in female patients between their thirties and fifties [5].

A correct identification of inner ear involvement in patients with systemic autoimmune diseases is essential for the possibility of near-complete hearing restoration with appropriate treatment [9]; however, it is often misdiagnosed due to variable clinical presentation, limited knowledge, sparse evidence, and lack of specific diagnostic tests.

The aim of this review is to analyse the available evidence of the different clinical presentations of audiological and vestibular symptoms in systemic autoimmune diseases, although this is often only reported in the form of case reports due to the rarity of some of these conditions.

\section{Inner Ear Involvement in Autoimmune Diseases}

2.1. Pathophysiology of Inner Ear Involvement in Autoimmune Diseases. Pathophysiology of inner ear involvement in systemic autoimmune diseases is still unclear and may be related to circulating antibodies against a number of inner ear antigens leading to antibody-dependent cellmediated cytotoxicity, the activation of the complement system, a direct action of cytotoxic T cells, or immune complexmediated damage $[5,7,8,10-14]$.

The immune complex deposition seems to play a central role in inner ear involvement; it leads to vasculitis of inner ear vessels that determines atrophy of the stria vascularis and SNHL. The deposition of immune complexes reduces the calibre of the auditory arteries with a consequent decrease in blood flow; blood flow reduction induces an oxygen deficit that increases the ROS level responsible for damage to hair cells and spiral ganglion [15-17]. This pathogenic mechanism appears to be the major factor involved in cochlear and vestibular damage in systemic autoimmune diseases, especially when affecting the labyrinthine artery, the common trunk of the inner ear vascularization system [18].

Temporal bone studies clearly explain the mechanism of damage. The vascular ischemia, underlying vasculitis, initially determines the atrophy of the stria vascularis and hair cell death; the progression of the inflammation initiates bone inflammatory processes such as necrosis or cochlear fibrosis [8], more evident in the final stages of the disease [19].

\subsection{Audiovestibular Symptoms in Systemic Autoimmune} Diseases. Audiovestibular dysfunction in systemic autoimmune diseases may have different clinical presentations with high interindividual variability [20]. Hearing loss is the most common condition, followed by tinnitus and vertigo [8].

Characteristics of hearing impairment are extremely variable. The hearing loss is typically sensorineural, affecting mainly high frequencies $[8,9]$, although low-frequency and mid-frequency hearing loss are common in cases of vasculitis [20]. The general pattern of SNHL is rapidly progressive over a period of weeks to months, with great timing variability among different systemic diseases [21]; fluctuations in hearing are common, although the overall course is a progressive deterioration of auditory function [9]. Hearing loss is mainly bilateral and asymmetric; however, cases of unilateral SNHL that manifests in the contralateral ear after a variable time have been described [21]. In some cases, a temporary and acute blood flow reduction in the inner ear may be related with the onset of sudden sensorineural hearing loss (SSNHL), with complete or partial recovery after restoration of normal perfusion. SSNHL is common in patients with inner ear involvement following systemic autoimmune diseases, and may be the presenting symptom in some cases $[5,20]$. Despite hearing loss being mainly sensorineural, autoimmune diseases can also induce a conductive hearing loss (CHL). In these cases, CHL may follow the effusions of the middle ear and the inflammation of Eustachian tube mucosa or involvement of the ossicular chain [22-24].

Tinnitus in systemic autoimmune diseases is mainly found in association to hearing loss. It has been established that the decrease of peripheral input following hearing loss can trigger neuroplastic reactions up to the auditory cortex responsible for the onset of tinnitus. Therefore, it is probable that peripheral auditory dysfunction could initiate central changes that eventually lead to tinnitus onset in patients with autoimmune diseases [25-28].

Vestibular symptoms, such as rotational vertigo or disequilibrium, may follow temporary occlusion of the labyrinthine or the anterior vestibular artery $[1,11]$, and they often mimic primary inner ear disorders such as Menière's disease [3, 21].

A list of systemic autoimmune diseases that have been reported to be associated to audiovestibular symptoms, along with relevant literature references, is shown in Table 1.

2.2.1. Systemic Lupus Erythematosus. Systemic lupus erythematosus (SLE) is an autoimmune disease with multiorgan involvement and an incidence higher in women (82\%-96\%) than in men (4\%-18\%) [29].

The audiovestibular symptoms that are present in patients with SLE may follow antibody/antigen direct reactions, cytotoxic action, or immune complex deposition [15]. Vasculitis following immune complex deposition is the major cause of cochlear and vestibular damage in SLE patients. Immune complexes deposit in the auditory artery reducing the vessel calibre with a consequent decrease in blood flow and oxygen deficit; this stimulates the release of ROS that damage the hair cells and the spiral ganglions with consequent hearing impairment [16].

SNHL is the most common audiovestibular symptom in SLE, with a prevalence of between $6 \%$ and $70 \%$ [15]. Hearing loss may be bilateral or unilateral, slowly progressive, or sudden; it mainly affects high frequencies, mimicking the typical presbycusis pattern, but may also involve low and middle frequencies [15]. Maciaszczyk et al. [30] and Roverano et al. [18] described progressive, bilateral SNHL involving high frequencies. Khalidi et al. [31] reported unilateral SNHL involving mid and high frequencies (500, 1000, 2000, and $3000 \mathrm{~Hz}$ ) associated with a $16 \%$ word discrimination score as demonstrated by speech audiometry. Sperling et al. [32] described both bilateral and slowly progressive SNHL and unilateral SSNHL in patients with SLE. 
TABLE 1: Systemic autoimmune diseases associated to audiovestibular symptoms.

\begin{tabular}{|c|c|c|c|}
\hline Autoimmune disease & Prevalence of audiovestibular involvement & Classification & Literature reference \\
\hline Systemic lupus erythematosus & $6-70 \%$ & $\begin{array}{l}\text { Systemic autoimmune } \\
\text { rheumatic disorders }\end{array}$ & {$[15,16,18] ;[29-37] ;[38]$} \\
\hline Cogan syndrome & $31-45 \%$ & Systemic vasculitis & [39-44]; [45] \\
\hline Sarcoidosis & $5-96 \%$ & Systemic granulomatous diseases & {$[46-50] ;[51]$} \\
\hline Rheumatoid arthritis & $25-72 \%$ & $\begin{array}{c}\text { Systemic autoimmune rheumatic } \\
\text { disorders }\end{array}$ & {$[24] ;[52-54]$} \\
\hline Antiphospholipid syndrome & Case reports only & $\begin{array}{l}\text { Autoimmune hypercoagulable } \\
\text { condition }\end{array}$ & {$[55-60]$} \\
\hline Polyarteritis nodosa & Case reports only & Systemic vasculitis & {$[61-70] ;[71]$} \\
\hline Behcet's disease & $12-80 \%$ & Systemic vasculitis & {$[72-77] ;[45]$} \\
\hline Takayasu's arteritis & Case reports only & Systemic vasculitis & {$[78-85] ;[79] ;[86]$} \\
\hline Relapsing polychondritis & $40-54 \%$ & $\begin{array}{l}\text { Autoimmune connective } \\
\text { tissue disorder }\end{array}$ & {$[23] ;[87-92] ;[93]$} \\
\hline Wegener's granulomatosis & $8-65 \%$ & Systemic vasculitis & [94-97] \\
\hline Susac's syndrome & Case reports only & Systemic vasculitis & [98-102]; [103] \\
\hline Sjögren's syndrome & $22-46 \%$ & $\begin{array}{l}\text { Systemic autoimmune } \\
\text { rheumatic disorders }\end{array}$ & [22]; [104-109]; [110] \\
\hline Myasthenia gravis & $22-34 \%$ & $\begin{array}{c}\text { Autoimmune condition affecting } \\
\text { neuromuscular junction }\end{array}$ & [111-119]; [120] \\
\hline Multiple sclerosis & $1-28 \%$ & $\begin{array}{l}\text { Autoimmune inflammatory } \\
\text { demyelinating disease }\end{array}$ & {$[4,15] ;[121-129] ;[130]$} \\
\hline Hashimoto thyroiditis & Case reports only & Autoimmune thyroid disease & {$[131]$} \\
\hline Mixed cryoglobulinemia & $22 \%$ & Systemic vasculitis & {$[132]$} \\
\hline Giant cell arteritis & $7-100 \%$ & Systemic vasculitis & {$[133-135]$} \\
\hline Vogt-Koyanagi-Harada’s disease & $48-62 \%$ & Systemic granulomatous diseases & {$[136]$} \\
\hline Ulcerative colitis & $2-5 \%$ & $\begin{array}{c}\text { Autoimmune inflammatory } \\
\text { bowel disease }\end{array}$ & {$[137,138]$} \\
\hline
\end{tabular}

Summary of systemic autoimmune diseases that have been reported to be associated to audiovestibular symptoms, along with reported prevalence of audiovestibular involvement, classification, and relevant references.

Tinnitus is often associated with hearing loss in SLE, most probably following peripheral deafferentation [33-35].

The vestibular system could be involved in SLE, but vertigo and dizziness have rarely been reported $[35,36]$. Balance disorders as a consequence of SLE have been observed also in children [37]; however, the incidence of vestibular symptoms may be underestimated due to their slowly progressive onset and consequent compensation by the somatosensory system and vision.

2.2.2. Cogan's Syndrome. Cogan's syndrome (CS), first described in 1934, is a rare autoimmune disorder characterized by ocular and audiovestibular symptoms [39]. CS develops in young adults, mostly during their first three decades of life [40, 41]. The origin of CS is still unclear. Antibodies against Cogan peptide have been found in serum of patients with CS. Also, this peptide antigen shares sequence homology with CD148 and connexin 26, both involved in congenital deafness [42].

CS includes a large spectrum of clinical manifestations. Haynes et al. [43] defined two types of CS, a typical and an atypical variant. Typical CS is defined by ocular symptoms, classically presenting as nonsyphilitic interstitial keratitis
(IK), audiovestibular symptoms similar to those of Menière's disease (recurrent episodes of hearing loss, tinnitus, and vertigo), and an interval between the onsets of ocular and audiovestibular manifestations of less than 2 years. Atypical CS is characterized by different inflammatory ocular manifestations, with or without IK; audiovestibular symptoms (usually progressive hearing loss); and, most important, a delay of more than 2 years between the onset of ocular and audiovestibular manifestations. In many cases, it is difficult to differentiate between the two types of CS because some patients do not present IK at the onset of the disease or, alternatively, they develop this condition during the following years. Systemic manifestations are much more frequent in atypical CS and can be used in the differential diagnosis between the two types [41].

Inner ear involvement in CS has been reported with a prevalence of between $31 \%$ and $45 \%$ [40-44]. The most common audiovestibular manifestations in CS are hearing loss, vertigo, tinnitus, ataxia, and oscillopsia [41]. These symptoms can appear at any time during the course of the disease [44]. Hearing loss may be both unilateral and bilateral, often presenting as SSNHL with fluctuations or progressive worsening over time. Progression to complete bilateral hearing 
loss has been reported in almost $50 \%$ of patients during the follow-up period, whereas permanent hearing loss in one ear was observed in $20 \%$ of patients [44].

Tinnitus usually follows auditory deafferentation [41]. Abnormal vestibular function is found in $90 \%$ of patients with CS; at least $20 \%$ of the patients present spontaneous or gaze-induced nystagmus. Rarely, patients show clinical symptoms of vestibulopathy that last for days or weeks from the time of onset without spontaneous resolution that frequently results to hospitalization [44].

2.2.3. Sarcoidosis. Sarcoidosis is an inflammatory multisystem disease with unknown origin. CNS involvement is reported in about $5-7 \%$ of patients with systemic sarcoidosis, called neurosarcoidosis (NS) [46].

A cranial nerve neuropathy affecting the facial and optic nerves is a common finding in up to $80 \%$ of NS patients $[46,47]$.

Audiovestibular involvement is common in sarcoidosis. In a review of 50 patients with NS [48], a high incidence of audiovestibular manifestations was noted. Hearing loss was present in $49 / 50$, unilateral in $25 \%$ and bilateral in $75 \%$ of the patients. Tinnitus was reported in 30 patients $(61 \%)$, and vestibular impairment was recorded in $32 / 50$ (64\%) including vertigo, dizziness, and benign paroxysmal positional vertigo. A complete vestibular function testing was performed in 24 patients and found abnormalities in 23 (96\%). Of those, six (25\%) had unilateral alterations, 16 (67\%) had bilateral alterations, and one (8\%) had a nonlocalizing dysfunction. In another review, Babin [49] reported SNHL in approximately $90 \%$ of reported cases, characterized by sudden or rapidly progressive onset, and vestibular symptoms with abnormal vestibular functioning tests in a similar percentage of cases. In almost $50 \%$ of the cases, at least partial hearing recovery was achieved after high-dose systemic steroid administration, while balance disorders recovered either spontaneously or after treatment [49].

A recent study [50] reported two new cases of SSNHL due to probable NS, each having a quite different clinical course. In one case, unilateral SSNHL and facial palsy were the presenting symptoms of NS, while in the second, unilateral SSNHL occurred despite ongoing immunosuppressive treatment for NS.

2.2.4. Rheumatoid Arthritis. Rheumatoid arthritis (RA) is a chronic, inflammatory disease affecting nearly $0.6 \%$ of the general population [52]. Principal symptoms referred by patients are articular and periarticular, although RA can involve other organs including the heart, lung, skin, and eye [52].

Several events can lead to audiovestibular alterations during the course of RA; thus, a wide variation in the prevalence of different types of hearing impairment in RA patients may be found [53]. SNHL is the most common type of hearing impairment in RA patients ranging from $25 \%$ to $72 \%$ [53]. Conductive hearing loss and mixed hearing loss have also been reported, although less frequently [53].

A prospective case-control study [24] compared hearing disturbances in patients with RA with a control group. In
$60 \%$ of the RA patients, SNHL was observed and the difference was statistically significant at $500 \mathrm{~Hz}, 1 \mathrm{kHz}$, and $2 \mathrm{kHz}$ in both ears. CHL was reported in $17.1 \%$ of the RA patients compared to $5.7 \%$ of patients in the control group, with a statistically significant difference.

Pathogenesis of CHL in RA is still poorly understood; several hypotheses have been proposed. A laxity of the middle ear transducer mechanism [54] was proposed although other authors [24] suggested increased stiffness of the ossicular system.

2.2.5. Antiphospholipid Syndrome. The antiphospholipid syndrome (APS) is an acquired disorder characterized by the presence of antiphospholipid antibodies such as anticardiolipin (aCL) and lupus anticoagulant (LAC) antibodies causing hypercoagulability. The characteristic triad of the disease is the association of specific antibodies, arterial or venous thrombosis, and/or pregnancy morbidity and mortality [55]. APS is associated with microthrombosis, causing cutaneous manifestations like purpuric eruptions, livedo reticularis, and skin ulcerations. The involvement of the retina may cause amaurosis fugax [56].

The involvement of the inner ear has been reported in APS and may be related to antibodies targeting the small vessels of the labyrinthine circulation. Endothelial cells within the cochlear circulation might be activated by antiphospholipid antibodies directly or by inducing the formation of free radicals that, secondarily, damage the endothelium. These upregulated endothelial cells would initiate local microthrombus formation and subsequent ischemia to the target organ [57].

The association of aCL or LAC antibodies and SNHL was firstly reported by Naarendorp and Spiera [56] in six patients with SLE or a lupus-like syndrome. Toubi et al. [58] studied sudden and progressive SNHL in 30 patients showing that in the control group, no one had aCL antibodies, whereas $27 \%$ of the patient group had aCL antibodies in low to moderate concentration. In a subsequent study, Toubi et al. [59] reported that $31 \%$ of patients with idiopathic SSNHL were positive for aCL, compared with only $6 \%$ of matched control subjects. A prospective study [60] had 168 patients with progressive SNHL who underwent a screening panel of blood tests for autoimmune disease including aCL antibodies, anti-B2 glycoprotein, and LAC. In this population, fortytwo patients (25\%) had at least one elevated antiphospholipid antibody marker and twenty patients had two or more positive test results, suggesting that antiphospholipid antibodies could be involved in the pathogenesis of some forms of inner ear dysfunction, related to a microthrombus formation in the labyrinthine vasculature.

2.2.6. Polyarteritis Nodosa. Polyarteritis nodosa (PAN) is a systemic necrotizing vasculitis that mainly affects mediumsized arteries, although small arteries may also be involved [61]. The incidence of PAN ranges from 0 to 1.6 cases per million, and the prevalence of this disease is approximately 31 cases per million [62]. PAN affects men more frequently than women and occurs in all ethnic groups; the average age at onset is approximately 50 years [63]. 
The pathogenesis of idiopathic PAN remains enigmatic, although the clinical response to immunosuppressive therapy suggests that immunological mechanisms play an active pathogenic role. As in other forms of vasculitis, the presence of impaired endothelial function could reflect direct endothelial cell activation and damage resulting from primary inflammatory vasculitis or proinflammatory cytokines or antibodies [62].

Clinical manifestations of PAN include nonspecific constitutional manifestations, such as sickness, weight loss, fever, arthralgia, and myalgia. Dysfunction or damage of target organs may produce specific symptoms, often caused by occlusion or rupture of inflamed arteries. The most frequently involved territories are the skin and peripheral nervous system [62].

SNHL is often reported in PAN [64-66] and, in rare instances, may occur as the presenting symptom of the disease $[67,68]$. Hearing loss is typically bilateral and symmetrical, with sudden onset $[67,69]$ or a rapidly progressive course $[65,70]$. Alterations found in auditory brainstem responses (ABR) suggest an inner ear involvement, and the involvement of low frequencies may resemble those found in endolymphatic hydrops [69]. Tinnitus, vertigo, and occasional nausea and vomiting may also be found in patients with PAN [67].

2.2.7. Behcet's Disease. Behcet's disease (BD) is a chronic systemic relapsing syndrome affecting young adults and characterized by the presence of recurrent oral and genital ulcers, ocular inflammation, and skin lesions caused by a vasculitis involving small vessels [72].

Hearing loss is a common compliant in $\mathrm{BD}$, it is mainly bilateral and predominantly affects high frequencies; several studies have reported SNHL following cochlear impairment ranging from $12 \%$ to $80 \%$ in $\mathrm{BD}$ patients $[72,73]$. No relationship has been found between age or disease duration and inner ear involvement [73, 74]. A recent survey [75] of $65 \mathrm{BD}$ patients reported that audiovestibular complaints were found in $47 \%$ of patients. The most common symptoms were tinnitus (11\%), hearing loss (10\%), and vertigo (8\%); a case of unilateral SSNHL was also reported.

Nearly half of BD patients report orthostatic disequilibrium [72]. Studies of the vestibular function [73, 74] showed that $\mathrm{BD}$ mainly causes peripheral lesion rather than damage to the central vestibular tracts, although another study [76] showed a higher prevalence of central vestibular syndrome in BD patients. Magnetic resonance studies did not show any degenerative conditions of basal ganglia and brainstem atrophies in $\mathrm{BD}$ patients with abnormal vestibular function tests [75, 76]. Neural involvement in BD (neuro-Behcet) may appear with dizziness or vertigo as initial symptoms, mimicking a vestibular neuritis [77].

2.2.8. Takayasu's Arteritis. Takayasu's arteritis (TA), also known as aortitis syndrome, is a vasculitis that mainly affects large elastic arteries with symptoms caused by organ ischemia, aneurysm formation, and inflammation. TA is more prevalent in women of reproductive age, and clinical features usually reflect limb or organ ischemia that follow gradual stenosis of the involved arteries [78, 79].

The aetiology of hearing impairment in TA remains unknown [79]; it has been hypothesized that hearing loss follows the elevation of serum immune complexes that deposits in the inner ear or reversible circulatory disturbances with hypercoagulability in response to arterial disease [80]. Although TA involves medium and large calibre arteries, Noel et al. reported that the occlusion of small retinal vessels is a possible microcirculatory complication in TA; common immunopathology mechanisms with hearing loss could be hypothesized [81]. Moreover, Maruyoshi et al. speculated that hearing loss in TA could have a vascular background based on reversible circulatory disturbances due to vasculitis and/or some autoimmune pathogenesis in the inner ear, involving especially hair cells [80].

Only a few cases in the literature reported an association of TA with SNHL [82-84]. Hearing loss is often progressive, although it can be stable or fluctuating; is usually bilateral and asymmetric; develops over several weeks to months; and mainly involves high frequencies. SNHL may also present as a SSNHL. A good response to corticosteroid therapy has been reported for SNHL in TA, although it may also persist despite therapy [80]. Recently, Ralli et al. described a case of a 36-year-old woman with TA who had two episodes of SSNHL involving one ear at a time with an 11-month delay between each episode of treatment with hyperbaric oxygen therapy associated to corticosteroids, with significant improvements in both ears $[85,86]$.

2.2.9. Relapsing Polychondritis. Relapsing polychondritis (RP) is a rare connective tissue disorder affecting organs containing collagen, such as the eye, cartilage tissue, and skin. The diagnosis is based on clinical features and no specific test for this disease is available; thus, definitive diagnosis takes a long time, and often, the prognosis is poor [87]. Recurrent bouts of inflammation may lead to a permanent destruction of involved structures such as cartilage of the ears, nose, larynx, tracheobronchial tree, and cardiovascular system [88]. McAdam et al. [89] proposed diagnostic criteria for RP when three or more of the six clinical features are present: recurrent chondritis of both auricles; nonerosive, seronegative inflammatory polyarthritis; chondritis of the nasal cartilages; ocular inflammation (conjunctivitis, keratitis, scleritis, and uveitis); respiratory tract chondritis affecting laryngeal and tracheal cartilages; and cochlear and/or vestibular dysfunction (SNHL, tinnitus, and vertigo).

Auricular chondritis is a quite specific sign of RP. It is present in $20 \%$ of patients at the onset of the disease and in $90 \%$ during the course of the disease [90]. One or both ears can be affected. The ear concha is swollen, red, or less often purplish, hot, and painful even at the slightest contact. The ear lobe, which does not contain the cartilage, is spared.

Audiovestibular impairment is reported in $40-54 \%$ of all patients with RP [91]. These changes can represent the initial symptoms heralding the outbreak of the disease or appear after the onset of other symptoms. Typical manifestations can be bilateral or unilateral, are usually of sudden onset, and appear as perceptive deafness or tinnitus combined with 
or without vertigo and nausea [89]. CHL can be a result of a serous otitis following chondritis of the eustachian tube [23]. Further, the SNHL in RP patients has been suggested to be the result of inflammation of the internal auditory artery or its cochlear branch [92] or due to autoantibodies against the cochlea and vestibular organ [91]. This could explain the near-complete hearing recovery in patients after treatment with corticosteroids.

2.2.10. Wegener's Granulomatosis. Wegener's granulomatosis (WG) is an autoimmune disease of unknown aetiology characterized by necrotizing granulomatous inflammation of the respiratory tract, necrotizing glomerulonephritis, and systemic vasculitis that affects predominantly small vessels.

Although the pulmonary, nasal, and renal manifestations of WG are well described, hearing symptoms are less appreciated. Studies available in the literature report a prevalence of audiovestibular symptoms in $8 \%$ to $65 \%$ of patients with WG, mainly auditory symptoms with SNHL $[94,95]$ or CHL in cases of WG involving the middle ear [96]. Audiometric patterns of WG have been described as typically flat, although sometimes additional high frequency losses may coexist and differential diagnosis with noise exposure or age-related hearing loss may be difficult [96]. Hearing impairment may also present as SSNHL; Bakthavachalam et al. [97] identified that hearing loss was present in $56 \%$ of patients suffering from WG and SSNHL was the most common form, occurring in $47 \%$ of cases.

Hearing loss may also be a presenting symptom of WG. SNHL in WG is believed to be largely irreversible, therefore potentially adding to the patient's cumulative disability [96]. SNHL evaluation and monitoring are therefore recommended for appropriate patient management and could suggest a worsening of disease that may address to a specific treatment like cyclophosphamide rather than either methotrexate or azathioprine $[94,95]$.

2.2.11. Susac's Syndrome. Susac's syndrome (SS) is a rare immune disease characterized by encephalopathy, branch retinal artery occlusion, and SNHL [98]. SS has a higher prevalence in females, with a male: female ratio of $1: 3.5$ [99].

The pathophysiology of SS is not entirely clear. Antiendothelial cell antibodies (AECAs) in SS have been recently documented [99]. Potential targeted antigens suggested in studies focusing on AECAs include cytoskeletal proteins ( $\beta$-actin, $\alpha$-tubulin, and vimentin), glycolytic enzymes (glucose-3-phosphate-dehydrogenase and $\alpha$-enolase), and the prolyl-4-hydroxylase $\beta$ subunit, a member of the disulfide isomerase family [100].

Clinical manifestations of SS are thought to be caused by autoimmune-mediated occlusions of microvessels in the brain, retina, and inner ear that lead to a characteristic clinical trial of central nervous system (CNS) dysfunction [101]. At clinical onset, the most common manifestations are CNS symptoms, observed in two-thirds of patients, followed by visual symptoms and hearing disturbances. Unfortunately, only a small number of patients (around 13\%) show the characteristic symptoms of SS at disease onset; thus, definitive diagnosis is often delayed [101].

Hearing loss can be a dramatic and severely debilitating feature of SS; it may be mild and insidious or may be fluctuating, mimicking Menière's disease [102]. A loss of low or middle frequencies is typical, suggesting a vulnerability of the cochlear apex to microinfarction; loss of high frequencies can also occur [99]. Hearing loss often occurs overnight and may affect both ears. Dörr et al. refer to this clinical behaviour as the "bang-bang hearing loss" [101]. Severe hearing loss is often accompanied by vertigo and a roaring tinnitus; the occlusion of cochlear and vestibular arterioles may be the cause of these symptoms [99]. In these patients that often develop severe/profound SNHL and can no longer benefit from hearing aid amplification, cochlear implantation is a valid therapeutic option.

2.2.12. Sjögren's Syndrome. Primary Sjögren's syndrome (pSS) is a chronic autoimmune disease characterized by xerostomia and xerophthalmia due to lymphocyte infiltration of both salivary and lacrimal glands. It may also occur as a systemic disease involving the kidneys, lungs, liver, vessels, and lymph nodes. pSS mainly affects women in the fourth-fifth decade of life, and presenting symptoms are often oral and ocular [104]. In this context, autoantibodies to cardiolipin and M3 muscarinic receptors (mAchRs) in the serum of pSS patients are suspected to play a pathogenic role in the onset of progressive hearing loss and neurological complications [104].

Audiovestibular involvement in patients with pSS has been reported in the literature with a prevalence ranging from $22 \%$ to $46 \%$. Boki et al. [105] reported in a pSS patient the presence of SNHL affecting preferentially high frequencies. Tumiati et al. [106] reported SNHL in 46\% (14/30) of patients with pSS. Ziavra et al. [107] diagnosed SNHL in $22.5 \%(9 / 40)$ of pSS patients. Hearing loss as presenting complaint in pSS is quite uncommon and only limited to case reports [22]; SSNHL was recently reported in a 62-year-old female treated with high-dose methylprednisolone $(250 \mathrm{mg}$ ) infusion for 5 days with successful hearing restoration [108].

The high prevalence of cranial neuropathies is a known condition in pSS, mainly with trigeminal and facial nerve involvement [109].

Although pSS patients tend to have a higher prevalence of SNHL compared to the general population, no evidence of damage to the central auditory pathways was reported [106]. However, the prevalence of audiovestibular symptoms in pSS might be underestimated, suggesting that their association with pSS was not previously made because it had not been actively sought [106].

2.2.13. Myasthenia Gravis. Myasthenia gravis (MG) is the most common autoimmune disorder affecting the neuromuscular junction, characterized by muscle weakness and fatigue [111]. Weakness, the typical clinical symptom of MG, affects facial, ocular, bulbar, respiratory, or limb muscles and worsens after muscular activity [112].

In healthy subjects, acetylcholine (ACh), the primary neurotransmitter of the efferent auditory system, has been 
found to enhance the electromotility of outer hair cells (OHC) binding to acetylcholine receptors (AChRs), which are localized on the postsynaptic membrane of OHC [113].

In patients with $M G$, autoantibodies against $A C h R s$ were reported to bind with $\mathrm{AChRs}$ on $\mathrm{OHCs}$, inducing a progressive loss of AChRs that decreases OHC electromotility [114]. This cascade of events induces apoptosis in all three rows of OHCs, evolving into a clinically evident SNHL in rare cases [115]. The efferent auditory system has been investigated using contralateral acoustic stimulation (CAS) [116]. CAS produces physiological suppression of otoacoustic emissions [117] protecting the hair cells from noise of moderate to high intensity [118]. A reduced CAS effect has been reported in patients with MG compared to control subjects, suggesting a possible role of the progressive reduction of beta subunits of nicotinic AChRs associated to the destruction of the basal membrane and OHCs due to prolonged exposure to autoantibodies $[114,116]$.

At MG onset, patients do not refer hearing loss and pure tone audiometry (PTA) is often within normal range. A specific test studying, the activity of the OHC, otoacoustic emissions (OAE), may show some abnormalities, as OAE have been found to exhibit greater sensitivity to incipient cochlear damage compared to PTA, particularly for high frequencies $[115,119]$. A study on $16 \mathrm{MG}$ patients reported a clinical hearing loss in $30 \%$ of the patients, while $100 \%$ of the patients exhibited abnormal distortion product otoacoustic emissions (DPOAEs) and transient evoked otoacoustic emissions (TEOAEs) [114]. Therefore, OAE should always be performed in MG patients because they can early detect the MG-related effects on the ACh-innervated auditory system [111].

Additional audiological symptoms, such as tinnitus, should be always considered and investigated although seldom reported [27].

Vertigo, also reported in patients with MG, seems to be more related to musculoskeletal alterations rather than to vestibular impairment [112].

2.2.14. Multiple Sclerosis. Multiple sclerosis (MS) is traditionally considered an autoimmune inflammatory demyelinating disease of the central nervous system (CNS). The autoimmune pathogenesis of MS is still debated; recently, it has been hypothesized that it may be a homogeneous degenerative process analogous to primary neurodegenerative diseases [121]. As an exacerbating and remitting immune-mediated disorder of interfascicular oligodendrocyte-produced myelin, MS can impair acutely and transiently any CNS neural system, including the auditory pathways [122].

The evidence of a clear presence of macrophages in the human temporal bone of patients affected by autoimmune diseases [15] support the hypothesis that in MS, the autoimmunity mechanisms also affect the structures of the inner ear; hair cells and auditory and vestibular spiral ganglion neurons may be subject to the attack of lymphocytes, and their damage may present with SNHL and vertigo. The microglia, a cell population that belongs to the macrophage family and that is normally represented in the brain, has been shown to be active in aggressive forms of MS (phenotype M1). Temporal bone studies could suggest that microglia can migrate to the internal auditory canal and to the cochlea [4]. M1 microglia can demyelinate cochlear and vestibular structures causing SSNHL or vertigo; such episodes may be temporary due to the relapsing-remitting phases of MS that activates and inactivates the M1 microglia.

In the literature, several reports showed MS-related hearing deficits. Hearing loss may occur when MS involves both the peripheral and the brainstem auditory pathways [123]; however, in some case, MS lesions involving the auditory pathways may not determine a clinically evident hearing impairment [124]. In rare cases, SSNHL may be the only presenting symptom of MS and may appear early in the course of the disease with good prognosis and little or no residual hearing deficit [125].

MS patients typically report a difficulty in speech perception, especially in noise [126]. This alteration is due to an abnormal auditory processing, such as problems with dichotic listening tasks and auditory temporal processing [126]. Performance of chronic MS subjects in speech reception threshold (SRT) is normal in the standard clinical level $(70 \mathrm{~dB}$ above the SRT); however, when lower levels are used, performance significantly decreases compared to agematched controls suggesting a deficit in cognitive processing, such as attention and auditory discrimination, which is especially required in binaural integration of sound [127].

Furthermore, studies have shown that $40 \%$ to $55 \%$ of individuals with MS have at least an episode of dysarthria or speech alteration characterized by slowness, slurring, or difficulties in production or comprehension [127].

Disequilibrium in MS is often related to internuclear ophthalmoplegia, and multiple nystagmus constitutes the most typical vestibular signs of MS, although peripheral equilibrium may coexist [128]. Multidirectional nystagmus without latency may be an atypical central sign, and differential diagnosis with peripheral disorder, such as benign paroxysmal positional vertigo, can be more difficult, although adaptation and fatigue of nystagmus play a central role in differential diagnosis. When the clinical findings are not clear and ex-adjuvantibus criteria cannot be adopted, vestibular-evoked myogenic potential (VEMP) may be proposed for the differential diagnosis of positional vertigo in association with careful clinical history and otoneurologic examination [128].

Audiovestibular symptoms in young, neurologically normal subjects, especially when spontaneous recovery occurs, could represent an early sign of MS even when no demyelinated plaques are visible in the central nervous system; it would be recommended to evaluate these subjects with clinical, radiological, and electrophysiological tests to exclude peripheral incipient MS [129].

2.2.15. Other Autoimmune Conditions. Other autoimmune diseases associated to audiovestibular symptoms include Hashimoto's thyroiditis, mixed cryoglobulinemia, giant cell arteritis (GCA), Vogt-Koyanagi-Harada's disease, and Ulcerative Colitis.

Thyroid autoimmunity seems to affect the inner ear, particularly inducing hearing loss at lower frequencies [131]. 
In mixed cryoglobulinemia, unilateral SNHL has been found in $22 \%$ of patients following immune complex deposit in labyrinthine vessels determining both audiological and vestibular symptoms [132].

Hearing loss has been reported with a prevalence ranging from $7 \%$ to $100 \%$ in several case series of patients with GCA, a multisystemic vasculitis mainly involving largeand medium-sized blood vessels [133]. In a series of 44 patients with GCA, PTA at the time of diagnosis showed auditory dysfunction in all patients [134]. In some patients, hearing loss was progressive and appeared as an initial manifestation [135].

Bilateral rapidly progressive SNHL and tinnitus and vestibular manifestations have been observed in $48 \%$ to $62 \%$ of patients with Vogt-Koyanagi-Harada's disease [136].

In a retrospective study, SNHL was found in about $2 \%$ of patients with ulcerative colitis [137]. In these patients, OAE play a central role as they may indicate a cochlear involvement even when normal hearing thresholds are present [138].

2.3. Audiovestibular Diagnostic Workup in Systemic Autoimmune Diseases. The audiovestibular symptoms in systemic autoimmune diseases are often related to the entity of the autoimmune damage, as they may follow an inflammatory process in the inner ear or a direct macrophage aggression of the inner and-mainly-outer hair cells [15].

The current literature agrees that SNHL is the most common auditory symptom of systemic autoimmune diseases [5], but due to the different presentation forms (sudden or progressive) and severity (mild to severe) of SNHL, an early correlation between the symptom and the systemic autoimmune disease may be difficult. Furthermore, audiovestibular symptoms found in autoimmune conditions are also common to other conditions such as diabetes and hypertension. For these reasons, a correct differential diagnosis of the cause of the audiovestibular involvement is of utmost importance.

The diagnostic process in patients presenting with a variety of audiovestibular symptoms should begin with individual medical history and family history followed by traditional audiovestibular tests. The most important audiological test battery in all cases should include PTA possibly extended to the high-frequency region, TEOAE and DPOAE, and ABR [5, 129]. Vestibular testing should include a basic vestibular exam integrated with caloric test, video head impulse test, and VEMPs [129]. The aforementioned test batteries should be performed at the onset of the audiovestibular symptom and during follow-up to monitor the course of the disease. Audiovestibular examination should be integrated, when an autoimmune condition is suspected, with specific blood tests as summarized in Table 2 [139].

2.4. Treatment Approaches to Audiovestibular Symptoms in Systemic Autoimmune Diseases. The treatment of audiovestibular symptoms should first aim at preserving the function, such as hearing preservation and/or restoration in patients with SNHL, and then at solving disability, distress, and
TABle 2: Blood tests commonly used in patients with audiovestibular symptoms suggestive for a systemic autoimmune condition.

\begin{tabular}{lc}
\hline Test & Classification \\
\hline Red and white cell counts & General blood test \\
Coagulation test (aPTT, PT) & General blood test \\
Creatine kinase (CK) & General blood test \\
Alanine transaminase (ALT) & General blood test \\
Aspartate aminotransferase (AST) & General blood test \\
Erythrocyte sedimentation rate (ESR) & Inflammatory markers \\
C-reactive protein (CRP) & Inflammatory markers \\
Ferritin & Inflammatory markers \\
Enzyme-linked immunosorbent & Immunologic analysis \\
assay (ELISA) & Antibody \\
Rheumatoid Factor (RF) & Antibody \\
Anti-cyclic citrullinated peptide & Antibody \\
Antibody (CCP) & Antibody \\
Anti-double-stranded DNA & \\
(anti-dsDNA) & Antibody \\
Antiextractable nuclear antigen \\
(anti-ENA)
\end{tabular}

Summary of most relevant blood tests used to investigate a possible autoimmune condition.

quality of life. If an underlying autoimmune disease is suspected, treatment should be started after complete blood exams; in fact, steroid therapy, that is, commonly used as first-line treatment for SSNHL and other audiovestibular symptoms, may have an effect on the underlying autoimmune systemic disease and delays its diagnosis.

In patients with a diagnosis of systemic autoimmune disease, the treatment of the audiovestibular symptoms is usually strictly related to that of the systemic condition. Common treatment options for systemic autoimmune diseases that may present an audiovestibular involvement are summarized in Table 3.

The most common treatment for SSNHL is systemic or intratympanic administration of high doses of corticosteroids, associated with hyperbaric oxygen treatment in cases of SLE, APS, and TA $[85,86]$. Other associated treatments include antioxidant compounds to avoid progression of SNHL [142], hearing aids to support the residual hearing function, or cochlear implants in case of severe and profound SNHL [143, 144]. 
TABLE 3: Common treatment for systemic autoimmune diseases with audiovestibular involvement.

Disease
Systemic lupus erythematosus (SLE)
Cogan's syndrome (CS)
Sarcoidosis
Rheumatoid arthritis (RA)
Antiphospholipid syndrome (APS)
Polyarteritis nodosa (PAN)
Behcet's disease (BD)

Takayasu's arteritis (TA)

Relapsing polychondritis (RP)

Wegener granulomatosis (WG)

Susac syndrome (SS)

Sjögren's syndrome (pSS)

Myasthenia gravis (MG)

Multiple sclerosis (MS)
Treatment

Reference

SLE without major organ manifestations: antimalarials and/or glucocorticoids; nonsteroidal anti-inflammatory drugs may be used judiciously for limited periods of time in patients at low risk for drug-induced complications; in nonresponsive patients, immunosuppressive agents such as azathioprine, mycophenolate mofetil, and methotrexate should also be considered

Prednisone $1 \mathrm{mg} / \mathrm{kg} /$ day for two weeks and then tapered over 3 to 6 months; methotrexate for long-term treatment; alternative treatments are cyclophosphamide, azathioprine, tacrolimus, and rituximab

High dose of corticosteroids (20-40 mg/daily) for 6 to 18 months; high-dose intravenous n-methyl-prednisone with doses of up to $30 \mathrm{mg} / \mathrm{kg}$ for $1-5$ days has been commonly recommended for treatment of refractory neurosarcoidosis; in addition, methotrexate, azathioprine, and TNF-alpha antagonists

Methotrexate at disease onset (10-15 mg/week) and then $20 \mathrm{mg} /$ week for 4-8 weeks; it is possible to use prednisolone at high dosage $(40-60 \mathrm{mg})$ and tapering to $7.5 \mathrm{mg}$ at week 6 for a total of 12 weeks

Chronic treatment with low dose of acetylsalicylic acid

PAN without viral syndrome: prednisone $1 \mathrm{mg} / \mathrm{kg} /$ day and then tapering when remission is reached

Steroid treatment with azathioprine; for resistant cases, azathioprine + interferon + TNF- $\alpha$ antagonists

Prednisone $1 \mathrm{mg} / \mathrm{kg} /$ day; additionally, it is possible to use immunosuppressants such as methotrexate, azathioprine, mycophenolate mofetil, leflunomide, tacrolimus, and TNF-alpha antagonists

Corticosteroid treatment at high dosages; in addition, colchicine, methotrexate, azathioprine, intravenous immunoglobulins, minocycline, and leflunomide

Prednisone or equivalent $1 \mathrm{mg} / \mathrm{kg} /$ day, sometimes preceded in severe cases by intravenous methylprednisolone pulses $(7.5-15 \mathrm{mg} / \mathrm{kg} / \mathrm{day})$ for $1-3$ consecutive days; after two weeks, tapering with a decrease of $10 \%$ every two weeks for a total of 6 months; in case of long-term treatment (>2 years), $5 \mathrm{mg}$ /day; is also possible to use cyclophosphamide and rituximab for maintenance therapy

High-dosage corticosteroids; additionally, intravenous immunoglobulin, plasma exchange azathioprine, mycophenolate mofetil, methotrexate, cytochrome P450 enzymes, and cyclosporine A

Cyclosporine A for local treatment of eye disease; colchicine and steroid treatment are used; controversial use of rituximab

Immunosuppressant therapy; in addition, treatment with insulin,thyroid hormones, and pyridostigmine

Immunomodulating therapy: T cell suppressor (alemtuzumab, daclizumab); B-cell modulators (rituximab, ocrelizumab); unique anti-inflammatory agents (laquinimod); hormones (estriol); 3-hydroxy-3-methylglutaryl-coenzyme A reductase inhibitors; vitamin D

Treatment options for systemic autoimmune conditions, along with relevant references.

Tinnitus is commonly treated with approaches aimed to restore hearing function, with antidepressant drugs when a psychological involvement is detected, and using oral supplements that combine antioxidants and vasoactive substances [145].

Vertigo can be treated with high doses of corticosteroids [146] associated to betahistine, a strong antagonist of the histamine $\mathrm{H} 3$ receptor and a weak agonist of the histamine H1 receptor, that improves vascularization of the inner ear [145]. Additional therapeutic approaches include metoclopramide and antidepressant drugs (inhibitor of D1 receptor) that act on central function by reducing the sensation of vertigo, nausea, and gastrointestinal symptoms [146]. For chronic dizziness, specific rehabilitation treatments can be used to favor central vestibular compensation and restore normal balance function [147].

\section{Conclusion}

Audiovestibular symptoms may be found in a variety of autoimmune diseases, and diagnosis is essential to increase the chances of restoration when specific therapy is promptly initiated. Inner ear involvement in autoimmune diseases is ascertained by the history, clinical findings, an immunologic evaluation of the patient's serum, and response to immunosuppressive therapies, following exclusion of other known causes. 
Audiovestibular symptoms could play a role in the diagnostic process of autoimmune diseases as they may be an earlyonset symptom-and in some cases, the only symptom-of an autoimmune condition. Furthermore, they may be useful to monitor the progression of the systemic disease.

Systemic autoimmune diseases should always be considered in patients with audiovestibular symptoms such as progressive/fluctuating SNHL with no other explainable cause. When a systemic autoimmune disease involving the inner ear is suspected, predisposing factors must be investigated, such as noise exposure, ototoxic treatments, previous ear surgery, trauma, meningitis, or family history of hearing loss. The exclusion of concomitant conditions may be challenging, especially in the case of presbycusis- or noiseinduced hearing loss. The low prevalence of these conditions, the heterogeneity of studies available in literature, and the absence of randomized trials are the factors that limit the knowledge of inner ear involvement in systemic autoimmune diseases along with underestimation of the problem and consequent undertreatment.

\section{Conflicts of Interest}

The authors report no conflict of interest.

\section{References}

[1] A. J. Matsuoka and J. P. Harris, "Autoimmune inner ear disease: a retrospective review of forty-seven patients," Audiology \& Neuro-Otology, vol. 18, no. 4, pp. 228-239, 2013.

[2] E. Lehnhardt, "Sudden hearing disorders occurring simultaneously or successively on both sides," Zeitschrift für Laryngologie, Rhinologie, Otologie und ihre Grenzgebiete, vol. 37, no. 1, pp. 1-16, 1958.

[3] B. F. McCabe, "Autoimmune sensorineural hearing loss," The Annals of Otology, Rhinology, and Laryngology, vol. 88, no. 5, pp. 585-589, 1979.

[4] J. T. O’Malley, J. B. Nadol Jr, and M. J. McKenna, “Anti $\mathrm{CD} 163+$, Iba1+, and CD68+ cells in the adult human inner ear: normal distribution of an unappreciated class of macrophages/microglia and implications for inflammatory otopathology in humans," Otology \& Neurotology, vol. 37, no. 1, pp. 99-108, 2016.

[5] B. A. A. Rossini, N. O. Penido, M. S. L. Munhoz, E. A. Bogaz, and R. S. Curi, "Sudden sensorioneural hearing loss and autoimmune systemic diseases," International Archives of Otorhinolaryngology, vol. 21, no. 3, pp. 213-223, 2017.

[6] A. Vambutas and S. Pathak, "AAO: autoimmune and autoinflammatory (disease) in otology: what is new in immunemediated hearing loss," Laryngoscope Investigative Otolaryngology, vol. 1, no. 5, pp. 110-115, 2016.

[7] M. U. Malik, V. Pandian, H. Masood et al., "Spectrum of immune-mediated inner ear disease and cochlear implant results," The Laryngoscope, vol. 122, no. 11, pp. 2557-2562, 2012.

[8] M. J. Ruckenstein, “Autoimmune inner ear disease," Current Opinion in Otolaryngology \& Head and Neck Surgery, vol. 12, no. 5, pp. 426-430, 2004.

[9] T. Mijovic, A. Zeitouni, and I. Colmegna, "Autoimmune sensorineural hearing loss: the otology-rheumatology interface," Rheumatology, vol. 52, no. 5, pp. 780-789, 2013.
[10] B. Hervier, P. Bordure, A. Masseau, C. Calais, C. Agard, and M. Hamidou, "Auto-immune sensorineural deafness: physiopathology and therapeutic approach," La Revue de Médecine Interne, vol. 31, no. 3, pp. 222-228, 2010.

[11] A. Greco, A. de Virgilio, A. Gallo et al., "Idiopathic bilateral vestibulopathy: an autoimmune disease?," Autoimmunity Reviews, vol. 13, no. 10, pp. 1042-1047, 2014.

[12] A. Greco, M. Fusconi, A. Gallo, C. Marinelli, G. F. Macri, and M. de Vincentiis, "Sudden sensorineural hearing loss: an autoimmune disease?," Autoimmunity Reviews, vol. 10, no. 12, pp. 756-761, 2011.

[13] M. A. Jeffries and A. H. Sawalha, "Autoimmune disease in the epigenetic era: how has epigenetics changed our understanding of disease and how can we expect the field to evolve?," Expert Review of Clinical Immunology, vol. 11, no. 1, pp. 45-58, 2014.

[14] J. Kanzaki, "Immune-mediated sensorineural hearing loss," Acta Oto-Laryngologica, vol. 514, pp. 70-72, 1994.

[15] A. Di Stadio and M. Ralli, "Systemic lupus erythematosus and hearing disorders: literature review and meta-analysis of clinical and temporal bone findings," The Journal of International Medical Research, vol. 45, no. 5, pp. 1470-1480, 2016.

[16] M. Abbasi, Z. Yazdi, A. M. Kazemifar, and Z. Z. Bakhsh, "Hearing loss in patients with systemic lupus erythematosus," Global Journal of Health Science, vol. 5, no. 5, pp. 102-106, 2013.

[17] M. Ralli, D. Troiani, M. V. Podda et al., "The effect of the NMDA channel blocker memantine on salicylate-induced tinnitus in rats," Acta Otorhinolaryngologica Italica, vol. 34, no. 3, pp. 198-204, 2014.

[18] S. Roverano, G. Cassano, S. Paira et al., "Asymptomatic sensorineural hearing loss in patients with systemic lupus erythematosus," Journal of Clinical Rheumatology, vol. 12, no. 5, pp. 217-220, 2006.

[19] S. Kariya, Ö. Hizli, S. Kaya et al., "Histopathologic findings in peripheral vestibular system from patients with systemic lupus erythematosus: a human temporal bone study," Otology \& Neurotology, vol. 36, no. 10, pp. 1702-1707, 2015.

[20] C. Agrup and L. M. Luxon, "Immune-mediated inner-ear disorders in neuro-otology," Current Opinion in Neurology, vol. 19, no. 1, pp. 26-32, 2006.

[21] G. B. Hughes, B. P. Barna, S. E. Kinney, L. H. Calabrese, and N. J. Nalepa, "Clinical diagnosis of immune inner-ear disease," Laryngoscope, vol. 98, no. 3, pp. 251-253, 1988.

[22] M. Lidar, E. Carmel, Y. Kronenberg, and P. Langevitz, "Hearing loss as the presenting feature of systemic vasculitis," Annals of the New York Academy of Sciences, vol. 1107, no. 1, pp. 136-141, 2007.

[23] P. R. Cohen and R. P. Rapini, "Relapsing polychondritis," International Journal of Dermatology, vol. 25, no. 5, pp. 280-286, 1986.

[24] V. V. Raut, J. Cullen, and G. Cathers, "Hearing loss in rheumatoid arthritis," The Journal of Otolaryngology, vol. 30, no. 5, p. 289, 2001.

[25] L. E. Roberts, J. J. Eggermont, D. M. Caspary, S. E. Shore, J. R. Melcher, and J. A. Kaltenbach, "Ringing ears: the neuroscience of tinnitus," The Journal of Neuroscience, vol. 30, no. 45, pp. 14972-14979, 2010.

[26] J. J. Eggermont and L. E. Roberts, "The neuroscience of tinnitus," Trends in Neurosciences, vol. 27, no. 11, pp. 676-682, 2004. 
[27] A. Sheppard, S. H. Hayes, G. D. Chen, M. Ralli, and R. Salvi, "Review of salicylate-induced hearing loss, neurotoxicity, tinnitus and neuropathophysiology," Acta Otorhinolaryngologica Italica, vol. 34, no. 2, pp. 79-93, 2014.

[28] B. D. Auerbach, P. V. Rodrigues, and R. J. Salvi, "Central gain control in tinnitus and hyperacusis," Frontiers in Neurology, vol. 5, p. 206, 2014.

[29] C. Lin, S. W. Lin, S. F. Weng, and Y. S. Lin, "Risk of sudden sensorineural hearing loss in patients with systemic lupus erythematosus: a population- based cohort study," Audiology \& Neuro-Otology, vol. 18, no. 2, pp. 95-100, 2013.

[30] K. Maciaszczyk, T. Durko, E. Waszczykowska, A. ErkiertPolguj, and A. Pajor, "Auditory function in patients with systemic lupus erythematosus," Auris Nasus Larynx, vol. 38, no. 1, pp. 26-32, 2011.

[31] N. A. Khalidi, R. Rebello, and D. D. Robertson, "Sensorineural hearing loss in systemic lupus erythematosus: case report and literature review," The Journal of Laryngology and Otology, vol. 122, no. 12, pp. 1371-1376, 2008.

[32] N. M. Sperling, K. Tehrani, A. Liebling, and E. Ginzler, "Aural symptoms and hearing loss in patients with lupus," Otolaryngology and Head and Neck Surgery, vol. 118, no. 6, pp. 762-765, 1998.

[33] V. S. Dayal and M. H. Ellman, "Sensorineural hearing loss and lupus," The Journal of Rheumatology, vol. 26, no. 9, p. 2065, 1999.

[34] A. P. M. Gomides, E. J. do Rosário, H. M. Borges, H. H. M. Gomides, P. M. de Pádua, and P. D. Sampaio-Barros, "Sensorineural dysacusis in patients with systemic lupus erythematosus," Lupus, vol. 16, no. 12, pp. 987-990, 2007.

[35] E. Karatas, A. M. Onat, C. Durucu et al., "Audiovestibular disturbance in patients with systemic lupus erythematosus," Otolaryngology and Head and Neck Surgery, vol. 136, no. 1, pp. 82-86, 2016.

[36] A. Batuecas-Caletrío, J. del Pino-Montes, C. CorderoCivantos, M. I. Calle-Cabanillas, and J. A. Lopez-Escamez, "Hearing and vestibular disorders in patients with systemic lupus erythematosus," Lupus, vol. 22, no. 5, pp. 437-442, 2013.

[37] G. I. Gad and H. Abdelateef, "Function of the audiovestibular system in children with systemic lupus erythematosus," Current Allergy and Asthma Reports, vol. 14, no. 7, p. 446, 2014.

[38] G. Bertsias, J. P. A. Ioannidis, J. Boletis et al., "EULAR recommendations for the management of systemic lupus erythematosus. Report of a task force of the EULAR Standing Committee for International Clinical Studies Including Therapeutics," Annals of the Rheumatic Diseases, vol. 67, no. 2, pp. 195-205, 2008.

[39] R. F. Morgan and C. J. Baumgartner, “Menier's disease complicated by recurrent interstitial keratitis. Excellent result following cervical ganglionectomy," Western Journal of Surgery, vol. 42, pp. 628-631, 1934.

[40] V. D'Aguanno, M. Ralli, M. de Vincentiis, and A. Greco, "Optimal management of Cogan's syndrome: a multidisciplinary approach," Journal of Multidisciplinary Healthcare, vol. 11, pp. 1-11, 2018.

[41] A. Greco, A. Gallo, M. Fusconi et al., "Cogan's syndrome: an autoimmune inner ear disease," Autoimmunity Reviews, vol. 12, no. 3, pp. 396-400, 2013.

[42] C. Lunardi, C. Bason, M. Leandri et al., "Autoantibodies to inner ear and endothelial antigens in Cogan's syndrome," Lancet, vol. 360, no. 9337, pp. 915-921, 2002.
[43] B. F. Haynes, M. I. Kaiser-Kupfer, P. Mason, and A. S. Fauci, "Cogan syndrome: studies in thirteen patients, long-term follow-up, and a review of the literature," Medicine, vol. 59, no. 6, pp. 426-441, 1980.

[44] M. B. Gluth, K. H. Baratz, E. L. Matteson, and C. L. W. Driscoll, "Cogan syndrome: a retrospective review of 60 patients throughout a half century," Mayo Clinic Proceedings, vol. 81, no. 4, pp. 483-488, 2006.

[45] O. Singer, "Cogan and Behcet syndromes," Rheumatic Diseases Clinics of North America, vol. 41, no. 1, pp. 75-91, 2015.

[46] D. G. James and O. P. Sharma, "Neurological complications of sarcoidosis," Proceedings of the Royal Society of Medicine, vol. 60, no. 11, Part 1, pp. 1169-1170, 1967.

[47] F. G. Joseph and N. J. Scolding, "Neurosarcoidosis: a study of 30 new cases," Journal of Neurology, Neurosurgery, and Psychiatry, vol. 80, no. 3, pp. 297-304, 2009.

[48] I. B. Colvin, "Audiovestibular manifestations of sarcoidosis: a review of the literature," Laryngoscope, vol. 116, no. 1, pp. 75-82, 2006.

[49] R. W. Babin, C. Liu, and C. Aschenbrener, "Histopathology of neurosensory deafness in sarcoidosis," Annals of Otology, Rhinology \& Laryngology, vol. 93, no. 4, pp. 389-393, 1984.

[50] E. Cama, R. Santarelli, E. Muzzi et al., "Sudden hearing loss in sarcoidosis: otoneurological study and neuroradiological correlates," Acta Otorhinolaryngologica Italica, vol. 31, no. 4, pp. 235-238, 2011.

[51] R. P. Baughman and E. E. Lower, "Treatment of sarcoidosis," Clinical Reviews in Allergy and Immunology, vol. 49, no. 1, pp. 79-92, 2015.

[52] M. Takatsu, M. Higaki, H. Kinoshita, Y. Mizushima, and I. Koizuka, "Ear involvement in patients with rheumatoid arthritis," Otology \& Neurotology, vol. 26, no. 4, pp. 755761, 2005.

[53] L. Murdin, S. Patel, J. Walmsley, and L. H. Yeoh, "Hearing difficulties are common in patients with rheumatoid arthritis," Clinical Rheumatology, vol. 27, no. 5, pp. 637-640, 2008.

[54] J. N. Rosenberg, D. A. Moffat, R. T. Ramsden, W. P. Gibson, and J. B. Booth, "Middle ear function in rheumatoid arthritis," Annals of the Rheumatic Diseases, vol. 37, no. 6, pp. 522-524, 1978.

[55] W. A. Wilson, A. E. Gharavi, T. Koike et al., "International consensus statement on preliminary classification criteria for definite antiphospholipid syndrome: report of an international workshop," Arthritis and Rheumatism, vol. 42, no. 7, pp. 1309-1311, 1999.

[56] M. Naarendorp and H. Spiera, "Sudden sensorineural hearing loss in patients with systemic lupus erythematosus or lupus-like syndromes and antiphospholipid antibodies," The Journal of Rheumatology, vol. 25, no. 3, pp. 589-592, 1998.

[57] J. S. Levine, D. W. Branch, and J. Rauch, "The antiphospholipid syndrome," The New England Journal of Medicine, vol. 346, no. 10, pp. 752-763, 2002.

[58] E. Toubi, M. A. Khamashta, A. Panarra, and G. R. V. Hughes, "Association of antiphospholipid antibodies with central nervous system disease in systemic lupus erythematosus," The American Journal of Medicine, vol. 99, no. 4, pp. 397-401, 1995.

[59] E. Toubi, K. Halas, J. Ben-David, E. Sabo, A. Kessel, and M. Luntz, "Immune-mediated disorders associated with 
idiopathic sudden sensorineural hearing loss," The Annals of Otology, Rhinology, and Laryngology, vol. 113, no. 6, pp. 445449, 2016.

[60] D. A. Mouadeb and M. J. Ruckenstein, "Antiphospholipid inner ear syndrome," Laryngoscope, vol. 115, no. 5, pp. 879883, 2005.

[61] J. C. Jennette, R. J. Falk, P. A. Bacon et al., "2012 revised International Chapel Hill Consensus Conference Nomenclature of Vasculitides," Arthritis and Rheumatism, vol. 65, no. 1, pp. 1-11, 2013.

[62] A. de Virgilio, A. Greco, G. Magliulo et al., "Polyarteritis nodosa: a contemporary overview," Autoimmunity Reviews, vol. 15, no. 6, pp. 564-570, 2016.

[63] J. Hernández-Rodríguez, M. A. Alba, S. Prieto-González, and M. C. Cid, "Diagnosis and classification of polyarteritis nodosa," Journal of Autoimmunity, vol. 48-49, pp. 84-89, 2014.

[64] W. Y. Adkins and P. H. Ward, "Temporal bone showing polyarteritis nodosa, otosclerosis, and occult neuroma," Laryngoscope, vol. 96, no. 6, pp. 645-652, 1986.

[65] M. Wolf, J. Kronenberg, S. Engelberg, and G. Leventon, "Rapidly progressive hearing loss as a symptom of polyarteritis nodosa," American Journal of Otolaryngology, vol. 8, no. 2, pp. 105-108, 1987.

[66] A. Bomholt, J. Bjerre Knudsen, H. Permin, B. Tommerup, and J. Gormsen, "Profound sensorineural hearing loss in polyarteritis nodosa. An atypical case of Cogan's syndrome," Archives of Oto-Rhino-Laryngology, vol. 236, no. 1, pp. 53-58, 1982.

[67] J. M. Rowe-Jones, D. C. Macallan, and M. Sorooshian, "Polyarteritis nodosa presenting as bilateral sudden onset cochleovestibular failure in a young woman," The Journal of Laryngology and Otology, vol. 104, no. 7, pp. 562-564, 1990.

[68] G. Lake-Bakaar and D. D. Gibbs, "Polyarteritis nodosa presenting with bilateral nerve deafness," Journal of the Royal Society of Medicine, vol. 71, no. 2, pp. 144-147, 2018.

[69] K. Tsunoda, J. Akaogi, N. Ohya, and T. Murofushi, "Sensorineural hearing loss as the initial manifestation of polyarteritis nodosa," The Journal of Laryngology and Otology, vol. 115, no. 4, pp. 311-312, 2001.

[70] E. Peitersen and B. H. Carlsen, "Hearing impairment as the initial sign of polyarteritis nodosa," Acta Oto-Laryngologica, vol. 61, no. 1-6, pp. 189-195, 2009.

[71] L. Forbess and S. Bannykh, "Polyarteritis nodosa," Rheumatic Diseases Clinics of North America, vol. 41, no. 1, pp. 33-46, 2015.

[72] C. Evereklioglu, Y. Cokkeser, S. Doganay, H. Er, and A. Kizilay, "Audio-vestibular evaluation in patients with Behçet's syndrome," The Journal of Laryngology and Otology, vol. 115, no. 9, pp. 704-708, 2001.

[73] G. Gemignani, S. Berrettini, P. Bruschini et al., "Hearing and vestibular disturbances in Behçet's syndrome," The Annals of Otology, Rhinology, and Laryngology, vol. 100, no. 6, pp. 459463, 1991.

[74] L. Soylu, M. Soylu, B. Aydoğan, and C. Özsahinoğlu, "Hearing loss in Behçet's disease," The Annals of Otology, Rhinology, and Laryngology, vol. 104, no. 11, pp. 864-867, 1995.

[75] I. Kulahli, K. Balci, E. Koseoglu, I. Yuce, S. Cagli, and M. Senturk, "Audio-vestibular disturbances in Behcet's patients: report of 62 cases," Hearing Research, vol. 203, no. 1-2, pp. 28-31, 2005.
[76] A. Greco, A. de Virgilio, M. Ralli et al., "Behçet's disease: new insights into pathophysiology, clinical features and treatment options," Autoimmunity Reviews, vol. 17, no. 6, pp. 567-575, 2018.

[77] N. Yesilot, M. Shehu, O. Oktem-Tanor, P. Serdaroglu, and G. Akman-Demir, "Silent neurological involvement in Behçet's disease," Clinical and Experimental Rheumatology, vol. 24, 5, Supplement 42, pp. S65-S70, 2006.

[78] F. Alibaz-Oner, S. Z. Aydin, and H. Direskeneli, "Recent advances in Takayasu's arteritis," European Journal of Rheumatology, vol. 2, no. 1, pp. 24-30, 2015.

[79] G. Keser, H. Direskeneli, and K. Aksu, "Management of Takayasu arteritis: a systematic review," Rheumatology, vol. 53, no. 5, pp. 793-801, 2014.

[80] H. Maruyoshi, K. Toyama, S. Kojima et al., "Sensorineural hearing loss combined with Takayasu's arteritis," Internal Medicine, vol. 44, no. 2, pp. 124-128, 2005.

[81] N. Noel, N. Butel, P. le Hoang et al., "Small vessel involvement in Takayasu's arteritis," Autoimmunity Reviews, vol. 12, no. 3, pp. 355-362, 2013.

[82] T. J. Siglock and K. H. Brookler, "Sensorineural hearing loss associated with Takayasu's disease," Laryngoscope, vol. 97, no. 7, pp. 797-800, 1987.

[83] T. Kunihiro, J. Kanzaki, T. O-Uchi, and A. Yoshida, "Steroidresponsive sensorineural hearing loss associated with aortitis syndrome,” ORL, vol. 52, no. 2, pp. 86-95, 1990.

[84] T. Yasui and T. Yamasoba, "Acute sensorineural hearing loss associated with aortitis syndrome," Acta Oto-Laryngologica. Supplementum, vol. 127, Supplement 559, pp. 29-33, 2009.

[85] M. Ralli, A. Greco, V. Falasca et al., "Recovery from repeated sudden hearing loss in a patient with Takayasu's arteritis treated with hyperbaric oxygen therapy: the first report in the literature," Case Reports in Otolaryngology, vol. 2017, Article ID 3281984, 6 pages, 2017.

[86] M. Ralli, A. Greco, and M. De Vincentiis, "Hearing loss in Takayasu's arteritis: a role for hyperbaric oxygen therapy?," The Journal of International Advanced Otology, vol. 13, no. 3, pp. 417-418, 2017.

[87] C. J. Michet Jr., C. H. McKenna, H. S. Luthra, and W. M. O'Fallon, "Relapsing polychondritis. Survival and predictive role of early disease manifestations," Annals of Internal Medicine, vol. 104, no. 1, pp. 74-78, 1986.

[88] L. Longo, A. Greco, A. Rea, V. R. Lo Vasco, A. de Virgilio, and M. de Vincentiis, "Relapsing polychondritis: a clinical update," Autoimmunity Reviews, vol. 15, no. 6, pp. 539-543, 2016.

[89] L. P. McAdam, M. A. O'hanlan, R. Bluestone, and C. M. Pearson, "Relapsing polychondritis: prospective study of 23 patients and a review of the literature," Medicine, vol. 55, no. 3, pp. 193-215, 1976.

[90] J. Shimizu, H. Oka, Y. Yamano, K. Yudoh, and N. Suzuki, "Cutaneous manifestations of patients with relapsing polychondritis: an association with extracutaneous complications," Clinical Rheumatology, vol. 35, no. 3, pp. 781-3, 2016.

[91] W. J. Issing, D. Selover, and P. Schulz, "Anti-labyrinthine antibodies in a patient with relapsing polychondritis," European Archives of Oto-Rhino-Laryngology, vol. 256, no. 4, pp. 163-166, 1999.

[92] J. M. Damiani and H. L. Levine, "Relapsing polychondritisreport of ten cases," Laryngoscope, vol. 89, 6, Part 1, pp. 929-946, 1979. 
[93] F. K. Lekpa and X. Chevalier, "Refractory relapsing polychondritis: challenges and solutions," Open Access Rheumatology: Research and Reviews, vol. 10, no. 10, pp. 1-11, 2018.

[94] J. H. Stone and G. Wegener's Granulomatosis Etanercept trial research group, "Limited versus severe Wegener's granulomatosis: baseline data on patients in the Wegener's granulomatosis etanercept trial," Arthritis and Rheumatism, vol. 48, no. 8, pp. 2299-2309, 2003.

[95] D. Takagi, Y. Nakamaru, S. Maguchi, Y. Furuta, and S. Fukuda, "Otologic manifestations of Wegener's granulomatosis," Laryngoscope, vol. 112, no. 9, pp. 1684-1690, 2002.

[96] A. D. Kornblut, S. M. Wolff, and A. S. Fauci, "Ear disease in patients with Wegener's granulomatosis," Laryngoscope, vol. 92, no. 7, pp. 713-717, 1982.

[97] S. Bakthavachalam, M. S. Driver, C. Cox, J. H. Spiegel, K. M. Grundfast, and P. A. Merkel, "Hearing loss in Wegener's granulomatosis," Otology \& Neurotology, vol. 25, no. 5, pp. 833-837, 2004.

[98] J. O. Susac, J. M. Hardman, and J. B. Selhorst, "Microangiopathy of the brain and retina," Neurology, vol. 29, no. 3, pp. 313-316, 1979.

[99] A. Greco, A. de Virgilio, A. Gallo et al., "Susac's syndrome pathogenesis, clinical variants and treatment approaches," Autoimmunity Reviews, vol. 13, no. 8, pp. 814-821, 2014.

[100] C. M. Magro, C. B. Marsh, J. N. Allen et al., "The role of antiendothelial cell antibody-mediated microvascular injury in the evolution of pulmonary fibrosis in the setting of collagen vascular disease," American Journal of Clinical Pathology, vol. 127, no. 2, pp. 237-247, 2007.

[101] J. Dörr, S. Krautwald, B. Wildemann et al., "Characteristics of Susac syndrome: a review of all reported cases," Nature Reviews Neurology, vol. 9, no. 6, pp. 307-316, 2013.

[102] A. Greco, A. Gallo, M. Fusconi, C. Marinelli, G. F. Macri, and M. de Vincentiis, "Meniere's disease might be an autoimmune condition?," Autoimmunity Reviews, vol. 11, no. 10, pp. 731-738, 2012.

[103] M. García-Carrasco, C. Mendoza-Pinto, and R. Cervera, "Diagnosis and classification of Susac syndrome," Autoimmunity Reviews, vol. 13, no. 4-5, pp. 347-350, 2014.

[104] M. Tucci, C. Quatraro, and F. Silvestris, “Sjögren's syndrome: an autoimmune disorder with otolaryngological involvement," Acta Otorhinolaryngologica Italica, vol. 25, no. 3, pp. 139-144, 2005.

[105] K. A. Boki, J. P. Ioannidis, J. V. Segas et al., "How significant is sensorineural hearing loss in primary Sjögren's syndrome? An individually matched case-control study," The Journal of Rheumatology, vol. 28, no. 4, pp. 798-801, 2001.

[106] B. Tumiati, P. Casoli, and A. Parmeggiani, "Hearing loss in the Sjogren syndrome," Annals of Internal Medicine, vol. 126, no. 6, pp. 450-453, 1997.

[107] N. Ziavra, E. N. Politi, I. Kastanioudakis, A. Skevas, and A. A. Drosos, "Hearing loss in Sjögren's syndrome patients. A comparative study," Clinical and Experimental Rheumatology, vol. 18, no. 6, pp. 725-728, 2000.

[108] K. S. Kim and H. S. Kim, "Successful treatment of sensorineural hearing loss in Sjögren's syndrome with corticosteroid," The Korean Journal of Internal Medicine, vol. 31, no. 3, pp. 612-615, 2016.

[109] E. L. Alexander, “Neurologic disease in Sjögren's syndrome: mononuclear inflammatory vasculopathy affecting central/peripheral nervous system and muscle. A clinical review and update of immunopathogenesis," Rheumatic Diseases Clinics of North America, vol. 19, no. 4, pp. 869-908, 1993.

[110] C. P. Mavragani, A. Nezos, and H. M. Moutsopoulos, "New advances in the classification, pathogenesis and treatment of Sjogren's syndrome," Current Opinion in Rheumatology, vol. 25, no. 5, pp. 623-629, 2013.

[111] M. Ralli, G. Altissimi, A. di Stadio, F. Mazzei, R. Turchetta, and G. Cianfrone, "Relationship between hearing function and myasthenia gravis: a contemporary review," The Journal of International Medical Research, vol. 45, no. 5, pp. 1459$1465,2016$.

[112] D. Grob, E. L. Arsura, N. G. Brunner, and T. Namba, "The course of myasthenia gravis and therapies affecting outcome," Annals of the New York Academy of Sciences, vol. 505, pp. 472-499, 1987.

[113] L. Tóth, T. Rácz, P. Diószeghy, G. Répássy, and I. Lampé, "Otoacoustic emission in myasthenia gravis patients and the role of efferent activation," Hearing Research, vol. 126, no. 1-2, pp. 123-125, 1998.

[114] S. A. Hamed, A. M. Elattar, and E. A. Hamed, "Irreversible cochlear damage in myasthenia gravis - otoacoustic emission analysis," Acta Neurologica Scandinavica, vol. 113, no. 1, pp. 46-54, 2006.

[115] A. R. Fetoni, M. Garzaro, M. Ralli et al., "The monitoring role of otoacoustic emissions and oxidative stress markers in the protective effects of antioxidant administration in noiseexposed subjects: a pilot study," Medical Science Monitor, vol. 15, no. 11, pp. PR1-PR8, 2009.

[116] S. Di Girolamo, A. d'Ecclesia, N. Quaranta, A. Garozzo, A. Evoli, and G. Paludetti, "Effects of contralateral white noise stimulation on distortion product otoacoustic emissions in myasthenic patients," Hearing Research, vol. 162, no. 1-2, pp. 80-84, 2001.

[117] A. Moulin, L. Collet, and R. Duclaux, "Contralateral auditory stimulation alters acoustic distortion products in humans," Hearing Research, vol. 65, no. 1-2, pp. 193-210, 1993.

[118] D. M. Williams and A. M. Brown, "The effect of contralateral broad-band noise on acoustic distortion products from the human ear," Hearing Research, vol. 104, no. 1-2, pp. 127146, 1997.

[119] M. Ralli, A. di Stadio, A. Greco et al., "Development of progressive hearing loss and tinnitus in a patient with myasthenia gravis: an overlooked comorbidity?," Hearing, Balance and Communication, vol. 15, pp. 1-7, 2017.

[120] J. B. Andersen, J. F. Owe, A. Engeland, and N. E. Gilhus, "Total drug treatment and comorbidity in myasthenia gravis: a population-based cohort study," European Journal of Neurology, vol. 21, no. 7, pp. 948-955, 2014.

[121] P. K. Stys, "Multiple sclerosis: autoimmune disease or autoimmune reaction?," The Canadian Journal of Neurological Sciences, vol. 37, Supplement 2, pp. S16-S23, 2010.

[122] M. Furst and R. A. Levine, "Hearing disorders in multiple sclerosis," Handbook of Clinical Neurology, vol. 129, pp. 649-665, 2015.

[123] A. Di Stadio, L. Dipietro, M. Ralli et al., "Sudden hearing loss as an early detector of multiple sclerosis: a systematic review," European Review for Medical and Pharmacological Sciences, vol. 22, no. 14, pp. 4611-4624, 2018. 
[124] R. L. Doty, I. Tourbier, S. Davis et al., "Pure-tone auditory thresholds are not chronically elevated in multiple sclerosis," Behavioral Neuroscience, vol. 126, no. 2, pp. 314-324, 2012.

[125] A. Ozunlu, N. Mus, and M. Gulhan, "Multiple sclerosis: a cause of sudden hearing loss," Audiology, vol. 37, no. 1, pp. 52-58, 1998.

[126] C. G. Matas, S. L. . A. Matas, C. R. S. . Oliveira, and I. C. Gonçalves, "Auditory evoked potentials and multiple sclerosis," Arquivos de Neuro-Psiquiatria, vol. 68, no. 4, pp. 528-534, 2010.

[127] T. M. Klugman and E. Ross, "Perceptions of the impact of speech, language, swallowing, and hearing difficulties on quality of life of a group of South African persons with multiple sclerosis," Folia Phoniatrica et Logopaedica, vol. 54, no. 4, pp. 201-221, 2002.

[128] D. Alpini, D. Caputo, L. Pugnetti, D. A. Giuliano, and A. Cesarani, "Vertigo and multiple sclerosis: aspects of differential diagnosis," Neurological Sciences, vol. 22, no. 8, pp. S84-S87, 2001.

[129] A. Di Stadio and M. Ralli, "Inner ear involvement in multiple sclerosis: an underestimated condition?," Multiple Sclerosis Journal, vol. 24, no. 9, pp. 1264-1265, 2018.

[130] J. Derwenskus and F. D. Lublin, "Future treatment approaches to multiple sclerosis," Handbook of Clinical Neurology, vol. 122, pp. 563-577, 2014.

[131] A. Arduc, S. Isık, S. Allusoglu et al., "Evaluation of hearing functions in patients with euthyroid Hashimoto's thyroiditis," Endocrine, vol. 50, no. 3, pp. 708-714, 2015.

[132] S. Berrettini, C. Ferri, L. L. Civita et al., "Inner ear involvement in mixed cryoglobulinaemia patients," British Journal of Rheumatology, vol. 34, no. 4, pp. 370-374, 1995.

[133] M. Samson, M. Corbera-Bellalta, S. Audia et al., "Recent advances in our understanding of giant cell arteritis pathogenesis," Autoimmunity Reviews, vol. 16, no. 8, pp. 833-844, 2017.

[134] J. C. Amor-Dorado, J. Llorca, C. Garcia-Porrua, C. Costa, N. Perez-Fernandez, and M. A. Gonzalez-Gay, "Audiovestibular manifestations in giant cell arteritis: a prospective study," Medicine, vol. 82, no. 1, pp. 13-26, 2003.

[135] L. Loffredo, S. Parrotto, and F. Violi, "Giant cell arteritis, oculomotor nerve palsy, and acute hearing loss," Scandinavian Journal of Rheumatology, vol. 33, no. 4, pp. 279-280, 2009.

[136] S. Al Dousary, "Auditory and vestibular manifestations of Vogt-Koyanagi-Harada disease," The Journal of Laryngology and Otology, vol. 125, no. 2, pp. 138-141, 2011.

[137] G. Casella, D. Corbetta, M. Zolezzi et al., "Symptomatic sensorineural hearing loss in patients with ulcerative colitis," Techniques in Coloproctology, vol. 19, no. 12, pp. 729-731, 2015.

[138] M. Sagit, S. Guler, A. Karaman, M. Yasar, A. Emiroglu, and I. Ozcan, "Cochlear involvement in patients with ulcerative colitis," The Journal of Laryngology and Otology, vol. 130, no. 2, pp. 128-133, 2016.

[139] C. Castro and M. Gourley, "Diagnostic testing and interpretation of tests for autoimmunity," The Journal of Allergy and Clinical Immunology, vol. 125, 2, Supplement 2, pp. S238S247, 2010.

[140] J. M. Davis 3rd, E. L. Matteson, American College of Rheumatology, and European League Against Rheumatism,
"My treatment approach to rheumatoid arthritis," Mayo Clinic Proceedings, vol. 87, no. 7, pp. 659-673, 2012.

[141] C. Pagnoux and L. Guillevin, "Treatment of granulomatosis with polyangiitis (Wegener's)," Expert Review of Clinical Immunology, vol. 11, no. 3, pp. 339-348, 2015.

[142] C. G. Le Prell, P. M. Gagnon, D. C. Bennett, and K. K. Ohlemiller, "Nutrient-enhanced diet reduces noise-induced damage to the inner ear and hearing loss," Translational Research, vol. 158, no. 1, pp. 38-53, 2011.

[143] N. Quaranta, R. Bartoli, F. Giagnotti, F. D. Cuonzo, and A. Quaranta, "Cochlear implants in systemic autoimmune vasculitis syndromes," Acta Oto-Laryngologica. Supplementum, vol. 122, no. 5, pp. 44-48, 2009.

[144] P. Mancini, F. Atturo, A. di Mario et al., "Hearing loss in autoimmune disorders: prevalence and therapeutic options," Autoimmunity Reviews, vol. 17, no. 7, pp. 644-652, 2018.

[145] J. Savage and A. Waddell, “Tinnitus,” BMJ Clinical Evidence, vol. 20, 2014.

[146] M. Strupp and T. Brandt, "Diagnosis and treatment of vertigo and dizziness," Deutsches Ärzteblatt International, vol. 105, no. 10, pp. 173-180, 2008.

[147] C. M. Cabrera Kang and R. J. Tusa, "Vestibular rehabilitation: rationale and indications," Seminars in Neurology, vol. 33, no. 3, pp. 276-285, 2013. 


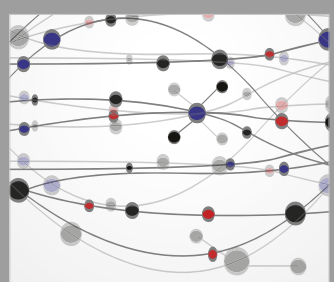

The Scientific World Journal
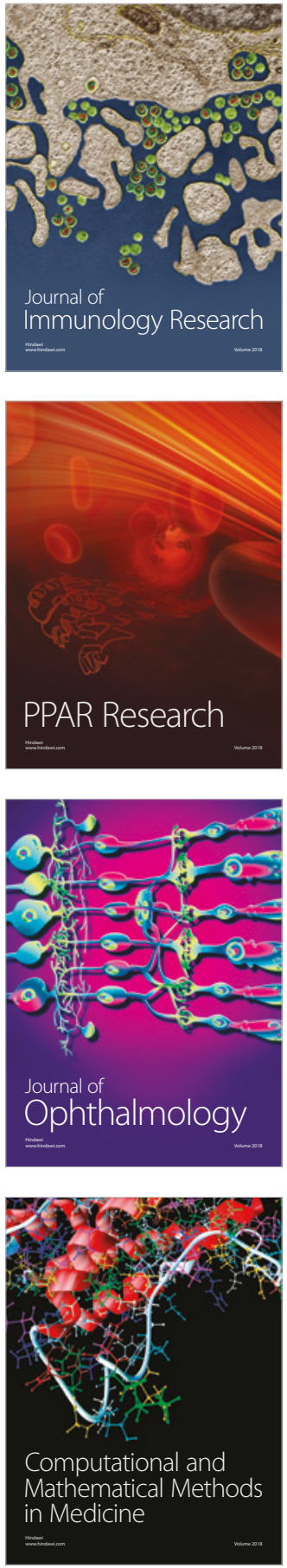

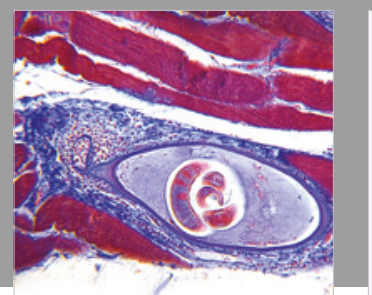

Gastroenterology Research and Practice

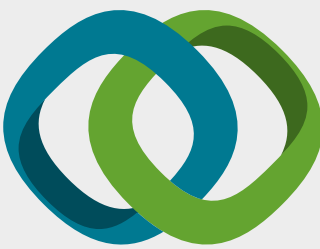

\section{Hindawi}

Submit your manuscripts at

www.hindawi.com
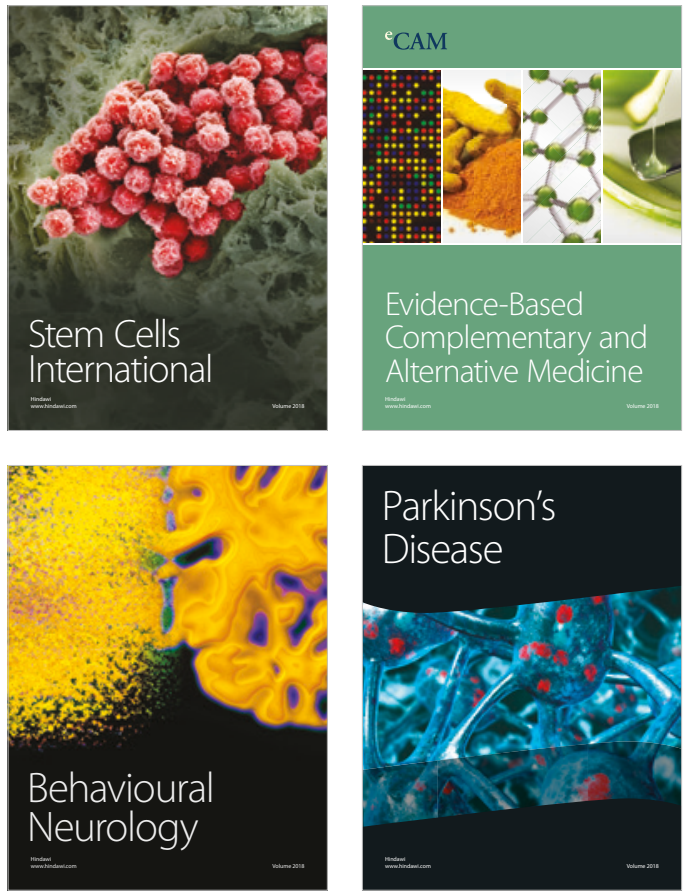

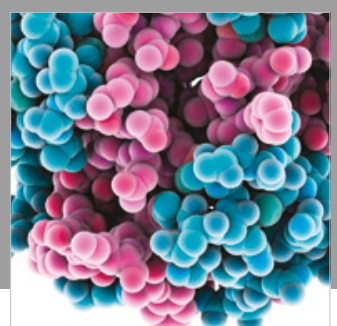

ournal of

Diabetes Research

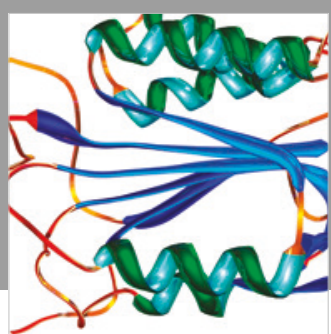

Disease Markers
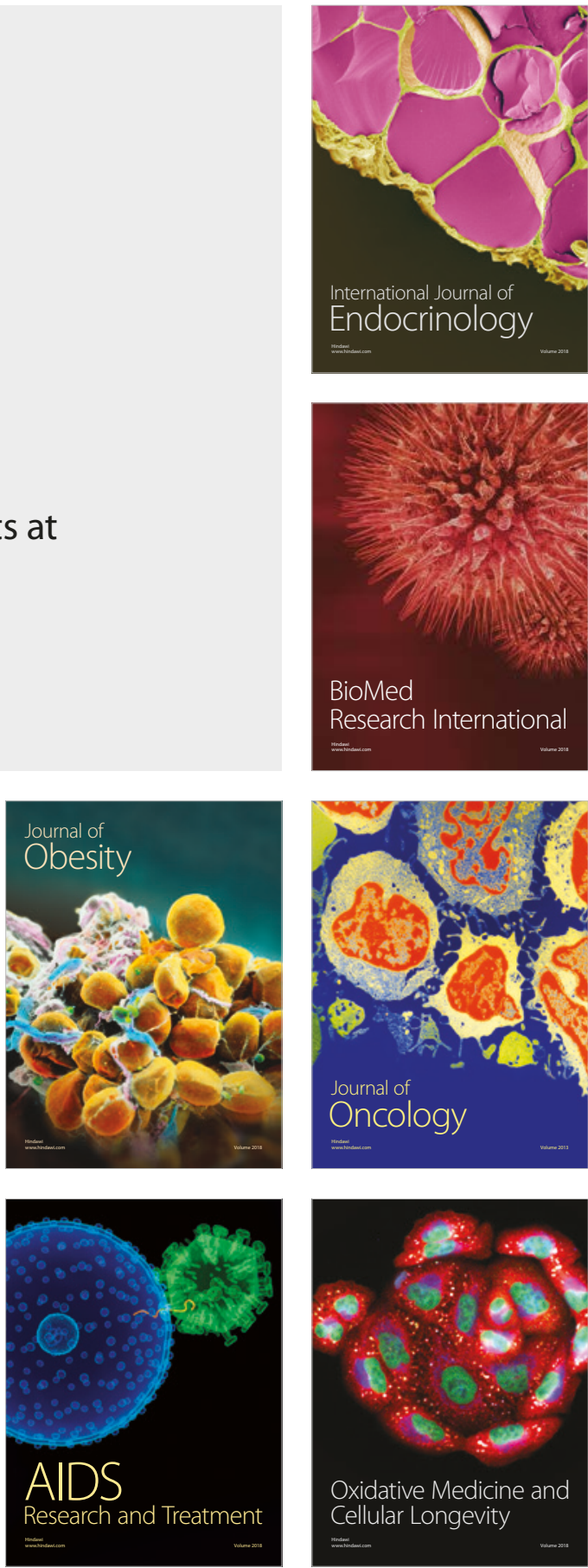